\title{
Living Donor Liver Transplant for Hepatocellular Carcinoma
}

\author{
Vijay Subramanian, MD, and William Chapman, MD \\ Department of Surgery, Section of Abdominal Organ Transplant, Washington University School of Medicine, St Louis, \\ MO
}

Hepatocellular carcinoma (HCC) is the most common cancer in the liver and is the third leading cause of cancerrelated deaths worldwide. Given the predilection of HCC in patients with underlying chronic disease, the majority of patients diagnosed with HCC are not resectable at the time of diagnosis.

Since publication of the 'Milan Criteria' in 1996, liver transplantation has been established as an effective treatment option for patients with unresectable HCC. ${ }^{1}$ The groups at the University of California, San Francisco (UCSF), ${ }^{2}$ along with our group at Washington University, ${ }^{3,4}$ proposed to expand this criteria to include patients outside of the Milan criteria with a larger tumor burden, and to maintain comparable outcomes.

In the US, many HCC patients have lower biologic MELD (Model for End-stage Liver Disease) scores, reflecting the status of their more compensated chronic liver disease. In the past, these patients would often await a donor organ while on the waitlist, and have progression of HCC prior to transplantation. Hence, to prioritize patients meeting the selection criteria, a MELD exception score would be granted. Starting in 2005, patients who had T2 disease (a solitary tumor $>2 \mathrm{~cm}$ but $<5 \mathrm{~cm}$, or up to three tumors, with the larges $<3 \mathrm{~cm}$ ) were given a MELD score of 22 , with upgrades of $10 \%$ priority every 3 months until transplant or dropout. Due to newer modeling predictions and concern that HCC patients were being overprioritized, the current allocation system applies a MELD score of 28 after a 6-month wait period for patients with HCC. In other

(C) Society of Surgical Oncology 2019

First Received: 25 January 2019;

Published Online: 1 April 2019

W. Chapman, MD

e-mail: chapmanw@wustl.edu regions of the world, various other methods are used to prioritize HCC patients for deceased donors, to make transplant a feasible option.

However, the average wait time for a liver transplant varies depending on the geographic location. Due to limitations in organ supply for deceased donors, the wait time can be prolonged and can result in the progression of disease, rendering patients non-transplantable. It is estimated that for each month on the waitlist, the dropout rate rises by $4 \%$ per month. ${ }^{5}$ In a study of 70 patients, Yao et al. estimated dropout rates of $7.2 \%, 37.8 \%$, and $55.1 \%$ at 6,12 , and 18 months, respectively. ${ }^{6}$

These important concerns regarding the availability of suitable organs as well as progression of disease while on the wait list have led to other strategic approaches for patients with HCC who are not candidates for liver resection. Bridging therapies prior to transplant, such as transarterial chemoembolization (TACE) and transarterial radioembolization (TARE) or external beam radiation, help control local disease progression. ${ }^{3}$ However, living donor liver transplantation now represents a viable source of transplantable organs and also has the advantage of being able to potentially reduce wait times.

In the current report, Wong et al. present their data comparing survival of patients receiving either living donor or deceased donor liver transplantation. ${ }^{7}$ This intention-totreat-based analysis represents single-center outcomes after transplantation in the study period, from 1995 to 2014. Overall, 188 patients were considered for living donor liver transplant (LDLT) and 187 were considered for deceased donor liver transplant (DDLT).

The dropout rate from the LDLT group was $14.4 \%$, which was thought to be mostly due to the lack of available donors. In the DDLT group, the dropout rate was up to $57 \%$. Among those patients who did not make it to transplant, $49.2 \%$ dropped out due to tumor progression and 
$45.9 \%$ due to liver decompensation. The wait time in the LDLT group was significantly shorter (24 days) compared with the DDLT group (250 days).

Overall survival from the time of listing was significantly better in the LDLT group compared with the DDLT group (1-, 3-, and 5-year overall survival rates of 94.1 vs. $77.5 \%, 81.4$ vs. $48.7 \%$, and 75.9 vs. $40.8 \%$, respectively). However, when a propensity score analysis was utilized to appropriately match recipients who make it to liver transplantation, the authors found no difference in both recurrence-free and overall survival after transplantation. This key finding suggests that the survival advantage from LDLT is likely in the population who dropped out from the waitlist of DDLT.

Although overall survival is better after LDLT, some earlier studies have suggested that LDLT had worse recurrence compared with after DDLT. It was postulated that this was likely due to the lack of ability to 'test tumor biology' during the waitlist time in patients receiving LDLT compared with those receiving DDLT.

It should also be noted that in some reports of LDLT for HCC, centers have offered LDLT for patients beyond the Milan/UCSF criteria. In the present study, $10.6 \%$ of patients (20/188) were outside of the UCSF criteria. In a multivariate analysis, tumor beyond UCSF was found to be an independent poor prognostic factor. This finding is echoed in another study from Bhangui et al., where tumor stage beyond the UCSF/Milan criteria showed a trend towards worse outcome. ${ }^{8}$ Our recommendation has been that patients beyond UCSF should undergo downstaging to meet the Milan criteria prior to liver transplant. ${ }^{4}$

Given these results, the Asian experience of LDLT for HCC has provided more insight into the appropriate selection of patients to maintain good outcomes. Studies from Korea and Japan have suggested expanding the criteria to include larger size (up to $6 \mathrm{~cm}$ ), as well as number of tumors (up to 7 or 10), providing comparable survival. ${ }^{9,10}$ Other histologic and biologic criteria have also been suggested to be included, such as $\alpha$-fetoprotein (AFP) levels to better prognosticate patients. ${ }^{11}$

Living donor liver transplantation is an important option to consider for increasing the donor pool, but at the same time it also opens up discussions of the utility and focus on the outcomes after transplantation, since the risks to the donor need to be justifiable. In 2012, the European Association of the Study of the Liver (EASL) suggested that LDLT should be considered for HCC in centers where the average wait time exceeds 6 months. Early experience from LDLT, and complications in donors, led to centers shying away from utilizing LDLT. This concern has diminished as experience builds and LDLT becomes the leading form of transplant in some centers across the world, especially when deceased donation is limited. As in other areas of medicine, patient selection remains one of the most important keys to success. In the appropriately selected patient, LDLT has the potential to offer comparable, if not better, patient outcomes compared with DDLT.

\section{REFERENCES}

1. Mazzaferro V, Regalia E, Doci R et al. Liver transplantation for the treatment of small hepatocellular carcinomas in patients with cirrhosis. N. Engl. J. Med. 1996; 334:693-699.

2. Yao FY, Ferrell L, Bass NM et al. Liver transplantation for hepatocellular carcinoma: expansion of the tumor size limits does not adversely impact survival. Hepatology. 2001; 33:1394-1403.

3. Chapman WC, Majella Doyle MB, Stuart JE et al. Outcomes of neoadjuvant transarterial chemoembolization to downstage hepatocellular carcinoma before liver transplantation. Ann. Surg. 2008;248:617-625.

4. Chapman WC, Garcia-Aroz S, Vachharajani N, et al. Liver transplantation for advanced hepatocellular carcinoma after downstaging without up-front stage restrictions. J. Am. Coll. Surg. 2017;224:610-621.

5. Llovet JM, Fuster J, Bruix J. Intention-to-treat analysis of surgical treatment for early hepatocellular carcinoma: resection versus transplantation. Hepatology. 1999;30:1434-1440.

6. Yao FY, Bass NM, Nikolai B, et al. A follow-up analysis of the pattern and predictors of dropout from the waiting list for liver transplantation in patients with hepatocellular carcinoma: implications for the current organ allocation policy. Liver Transpl. 2003;9:684-692.

7. Wong TCL, Ng KKC, Fung JYY, et al. Long-term survival outcome between living donor and deceased donor liver transplant for hepatocellular carcinoma: Intention-to-treat and propensity score matching analyses. Ann Surg Oncol. Epub 8 Feb 2019. https://doi.org/10.1245/s10434-019-07206-0.

8. Bhangui P, Vibert E, Majno P et al. Intention-to-treat analysis of liver transplantation for hepatocellular carcinoma: living versus deceased donor transplantation. Hepatology. 2011;53:1570-1579.

9. Takada $\mathrm{Y}$, Ito $\mathrm{T}$, Ueda M, et al. Living donor liver transplantation for patients with HCC exceeding the Milan criteria: a proposal of expanded criteria. Dig Dis. 2007;25:299-302.

10. Lee SD, Lee B, Kim SH et al. Proposal of new expanded selection criteria using total tumor size and (18)F-fluorodeoxyglucose - positron emission tomography/computed tomography for living donor liver transplantation in patients with hepatocellular carcinoma: The National Cancer Center Korea criteria. World J. Transplant. 2016;6:411-422.

11. Kim JM, Kwon CH, Joh JW, et al. Expanded criteria for liver transplantation in patients with hepatocellular carcinoma. Transplant Proc. 2014;46:726-729.

Publisher's Note Springer Nature remains neutral with regard to jurisdictional claims in published maps and institutional affiliations. 